\title{
"Fibromyalgia"- Diagnostic Concepts Rejuvenated: A Review
}

\section{Aghina Pradeep ${ }^{1}$, Vathsala Naik ${ }^{2}$, Gaurav ${ }^{3 *}$, Amandeep ${ }^{4}$, Sangeetha $^{4}$ and Bhagya Balakrishnan ${ }^{5}$}

${ }^{1}$ Final Year Postgraduate Student, Department of Oral Medicine and Radiology,

Bangalore Institute of Dental Sciences and Hospital, Bangalore, India

${ }^{2}$ Professor and Head, Department of Oral Medicine and Radiology, Bangalore

Institute of Dental Sciences and Hospital, Bangalore, India

${ }^{3}$ Assistant Professor, Department of Oral Medicine and Radiology, Bangalore

Institute of Dental Sciences and Hospital, Bangalore, India

${ }^{4}$ Reader, Department of Oral Medicine and Radiology, Bangalore Institute of

Dental Sciences and Hospital, Bangalore, India

${ }^{5}$ Post Graduate Student, Department of Oral Medicine and Radiology, Bangalore

Institute of Dental Sciences and Hospital, Bangalore, India

*Corresponding Author: Gaurav, Assistant Professor, Department of Oral Medicine and Radiology, Bangalore Institute of Dental Sciences and Hospital, Bangalore, India.

\author{
Received: October 21, 2021
}

Published: November 16, 2021

(C) All rights are reserved by Gaurav., et al.

\section{Abstract}

Fibromyalgia is a chronic disorder characterized by widespread musculoskeletal pain accompanied by fatigue, sleep, memory and mood issues. Although its etiopathogenesis and pathophysiology is known, their correlation as a diagnostic marker is poorly understood. As per WHO, it is estimated that up to three out of four people with the condition remain undiagnosed globally while the total prevalence stands approximately $0.20 \%$ amongst the Asian sub population.

As there is still a diagnostic dilemma in case of FM, various diagnostic criteria have been put forward since years. In the scenario of unpredictable cascade of the malady, various new diagnostic aids are being tried world-wide based on various governing factors including "Oxidative stress". This review describes the recent diagnostic criteria's and recent diagnostic advancement based on various molecular parameters, the thought process enveloping around 'Genetic' (coding of protein back bone and numerous functional groups, genetic signatures in coding genes for HLA, MHC etc.) and 'Metabolic' (blood and urine) components.

Although still in the phase of clinical trials, this unique technique of "Metabolic finger printing" holds a promising potential, not only towards turning a new leaf in the diagnostic approach of Fibromyalgia, but also determining a targeted therapy based treatment protocol, thereby bringing about a renaissance in this particular field.

Keywords: HLA; 5HT2A; Serotonin Receptors; LPC; Darapladib

\section{Introduction}

Fibromyalgia is a condition with unknown etiology, marked by Chronic widespread musculoskeletal pain with multiple symptoms, including TMDs, MPS, fatigue, sleep disturbances, cognitive dysfunction, and depressive episodes. It is believed to be a disorder of central pain processing, that produces hyperalgesia, allodynia etc.
It is diagnosed in $2.5 \%$ of Europeans and in $1.7 \%$ of the population in Asia. Global statistics reveal $24.6 \%$ of fibromyalgia patients seeing more than six health care professionals before reaching their diagnosis which heralds the diagnostic complexity of this condition. In the literature it has also been called as Fibromyalgia syndrome or Fibromyositis. There is a strong gender predilection towards females (F:M=9:1), hence the disease is also called as Woman's disease.

Citation: Gaurav., et al. ““Fibromyalgia”- Diagnostic Concepts Rejuvenated: A Review". Acta Scientific Dental Sciences 5.12 (2021): $45-49$. 
Patients suffering from this disease tolerate excruciating pain on a daily basis, and are on a panel of continuous emotional disturbances, so they are called as "Fibro warriors". As Oral Physicians, the occurrence of chronic pain disorders of the orofacial region which mimic FM are of great diagnostic challenge.

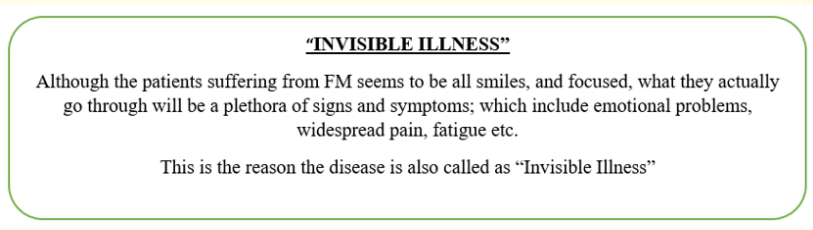

Figure a

Etiopathogenesis

Many a roadblock to diagnostic dilemma, without a reliable diagnostic criterion, leaves a very little hope for patients to lead a quality based life. Hence it is the necessity of the hour for certain confirmatory investigations and treatment modalities which could combat the challenges posed.

In this regard, the search for biomarkers aiding in diagnosis and early detection of FM is still a need of the hour and an ongoing process.

Numerous studies have explained FM in terms of, etiopathogenesis, as well as pathophysiology of the disease process. Some of the important studies included in literature which gives a near concrete hypothesis throwing a limelight on the disease have been listed below.

In 2006, Daniel J Claw., et al. from Chronic Pain and Fatigue Research Centre, Michigan, USA, published an article in American Journal of Medicine, named "Fibromyalgia: an overview" which states that specific genetic polymorphisms are associated with Fibromyalgia encoding 'Serotonin receptors, 5HT2A a Serotonin transporter, substance. $\mathrm{P}$ and Glutamate were all seen in an increased frequency in patients with FM while compared to control groups. The article also states that these individuals have a fundamental "wind-up" summation pain phenomenon which is a problem with central pain or afferent sensory processing rather than an abnormality confined to the region of the body where pain is experienced [1].

Yet another recent study was conducted by Chih-Hsein-Hung., et al. in 2020 (published in BMJ journal) showed activation of ac- id-sensing ion channel 3 by Lysophosphatidylcholine 16:0 (LPC) which mediates psychological stress-induced Fibromyalgia like pain. The study was conducted by inducing non-painful sound stimuli as psychological stress in animal model (mice). It was found that mice developed long-lasting non-inflammatory hyperalgesia after repeated and intermittent sound stress exposures. Although the study was conducted on an animal model, it positively demonstrated that the stress is directly proportional to the different symptoms causing FM [4]. The concepts they put forth to establish the relationship between stress and FM can be summarised as shown in figure 1.

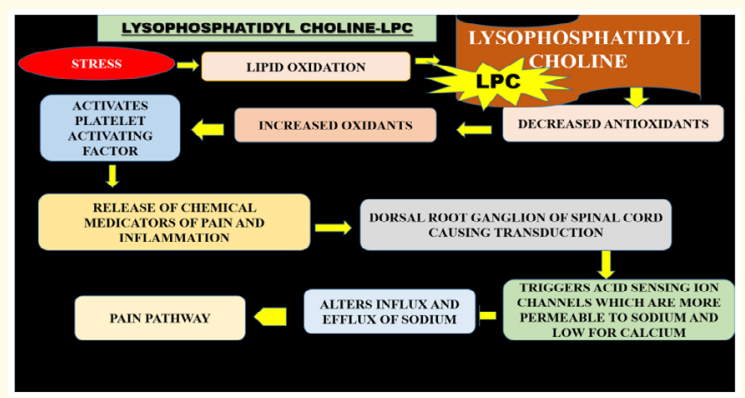

Figure 1

Alteration in influx and efflux of sodium ultimately results in cell polarisation, initiating the pain pathway causing Chronic hyperalgesia in case of FM. Clinical evidences showed that excessive LPC exists in FM cases and their expression was correlated with the pain symptoms in patients with high oxidative stress and disease severity. This correlation between Stress, LPC 16:0, and lipid oxidation was thought of a path breaking gateway to treat the checkmate of Fibromyalgia [4].

Yet another study conducted by Kevin V Hackshaw., et al. in 2021 highlighted the search for "Biomarkers" in Fibromyalgia, in which a Biomarker fingerprinting technique was hypothesised in serum using Infra-Red Micro spectroscopy. The spectroscopic findings showed significant increase in metabolites like: Hippuric, Succinic and Lactic acid, which are directly proportional to stress [3].

Hippuric acid causes Reactive Oxygen Species (ROS) accumulation thereby resulting in Oxidative stress, more predominantly seen in patients suffering from Chronic Kidney Disease (CKD) basically affecting the NRF2 pathway. Lactic acid on the other hand lays the concept of formation of trigger points in stressed conditions like that of FM and MPDS, usually occurring in conditions of an- 
aerobic glycolysis in which there is deficiency of Calcium release from sarcoplasmic reticulum which ultimately fails to break the actomyosin bridge resulting in a state of sustained knot like state of muscle contraction. Succinic acid similarly acts as an anxiolytic molecule which increases Serotonin level thereby resulting in Stressed conditions [3].

Kevin V Hackshaw., et al. conducted yet another study on 50 patients with FM, 29 with Rheumatoid arthritis, 19 with Osteoarthritis and 23 with Lupus. They collected blood sample from these patients and conducted Vibrational spectroscopy to differentiate patients with FM from others and found out that Protein backbones and Pyridine Carboxylic acid might serve as biomarkers in patients with Fibromyalgia and Vibrational Spectroscopy can be used as a reliable diagnostic tool for differentiating FM from others. In the available literatures, it has been shown that in the conditions of oxidative and Nitrosative stress, where there is more of oxides, peroxides, and super oxides, the cytoskeletal phenomena of Carboxylation, backbone cleavage, cross linking and unfolding along with cell signalling and scavenging processes shows marked reduction thereby accounting for the above-mentioned hypothesis [3].

Linkage studies confirmed the strong genetic contribution to FM and suggested linkage of FM to the chromosome $17 \mathrm{p} 11.2$ q11.2 regions. The large candidate gene study identified significant differences in allele frequencies between cases and controls for 3 genes: GABRB3, TAAR1, (genes that play a significant role in regulating the neurotransmission) and GBP1(associated with chronic EBV infection) [3].

\section{Role of stress in fibromyalgia}

Stress is one of the strong precipitating etiological factor. It could be in the form of physical, psychological or social causes. Various events related to these, are termed as stressors or triggers with concentrated areas of this called as trigger points.

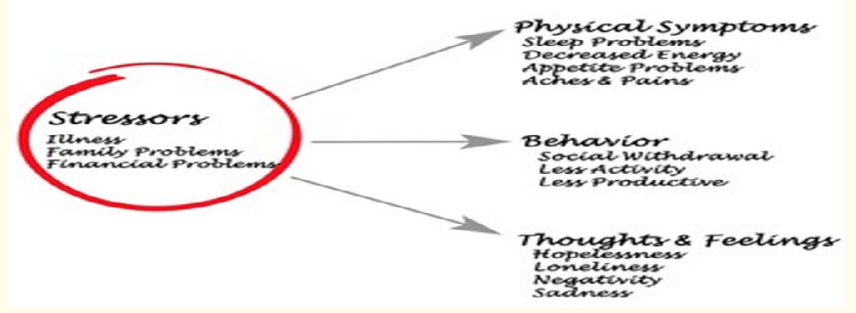

Figure 2
Trigger points are discrete, focal, hyperirritable spots located in a taut band of skeletal muscle. They produce pain locally and in a referred pattern and often accompany chronic musculoskeletal disorders. As per various studies there are approximately 18 trigger points as shown in figure 1 for Fibromyalgia.

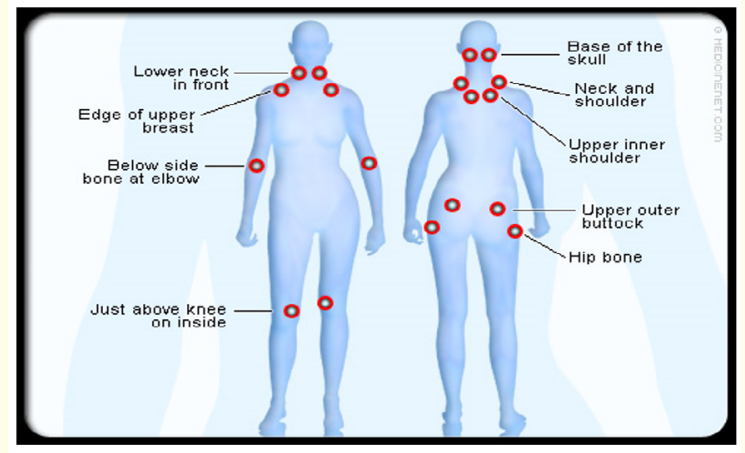

Figure 3

\section{Mechanism of stress}

Nervous system and immune system has an inbuilt biochemical language expressed through a homeostasis existing between their respective secreted hormones. Whenever the body perceives stress, cortisol (Stress hormone) is released into the blood stream. This suppresses and weakens the signals, which in turn regulates specific physiological neuroendocrine processes from the hormonal proteins like growth Hormone, Follicle stimulating hormone etc and cytokines like IL6. This delicate break in regulatory signals not reaching the neuroendocrine system, disturbs the body equilibrium which results in declined neural functions such as development, memory and wound healing and ultimately resulting in an altered and depressed life.

Along with this, a consequent central pain processing system alteration is also seen rendering Fibromyalgic symptoms as shown in figure 4 .

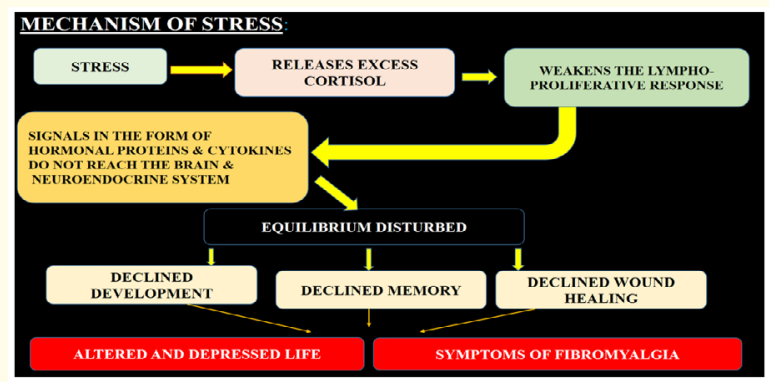

Figure 4 


\section{Diagnostic criteria}

According to the 1990 American College of Rheumatology (ACR Criteria); the criteria for diagnosis of FM include: History of chronic widespread pain $>/=3$ months, Patients must exhibit $>/=11$ out of 18 tender points [8].

\section{Diagnostic criteria of 2016}

The 2016 criteria require that patients have pain in 4 of 5 regions, called "generalized pain" to distinguish it from the 1990 definition of "widespread pain".

Revised diagnostic criteria

AAPT Diagnostic Criteria for Fibromyalgia-2019.

The criteria were divided in to 5 dimensions [11]:

- Dimension 1: Core diagnostic criteria

- Dimension 2: Common features

- Dimension 3: Common medical co-morbidities

- Dimension 4: Neurobiological, Psychosocial, and Functional consequences

- Dimension 5: Putative neurobiological and psychosocial mechanisms, risk factors.

Based on this, the proposed criteria for FM dimension 1 require $\geq 11$ pain sites be endorsed on the 35 -point body manikin.

But as the 35-point manikin is highly impractical for a diagnostic criterion, the appropriate sites were grouped together, and this resulted in a new body manikin that had only 9 defined sites [11].

This study reached in a new diagnostic criterion:

- $\quad$ Out of 9 pain points at least 6 or more should be positive.

- Moderate to severe sleep problems or Fatigue

- Multisite pain plus fatigue or sleep problems must have been present for at least 3 months (Figure 5) [11].

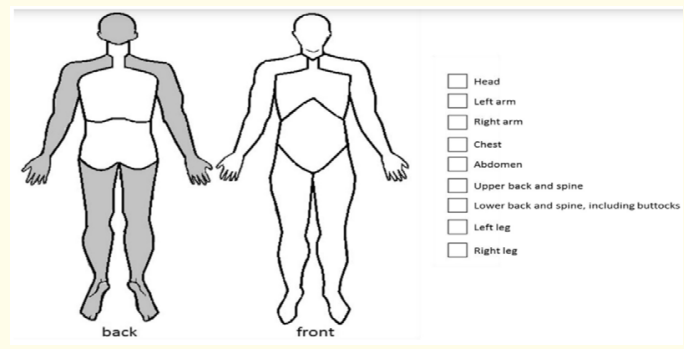

Figure 5

\section{Differential diagnosis}

According to the National Fibromyalgia and Chronic Pain Association, it is estimated that it takes around 5 years on an average for a symptomatic Fibromyalgia patient to actually receive an accurate diagnosis. The reason for this is that there are multiple diseases which mimic this condition leading to a diagnostic dilemma. To name a few are Lupus, Multiple Sclerosis, Rheumatoid Arthritis, Type 2 diabetes, Chronic Fatigue Syndrome etc.

Out of the various differential diagnoses which mimic Fibromyalgia (FM), Myofascial Pain Dysfunction Syndrome, today called as Myofascial Pain, is one of the most challenging feature masking the commonly associated symptoms of FM. Therefore, as a clinician and diagnostician, it is important on our part to know how to differentiate between the two entities. In Fibromyalgia, there is a presence of widespread muscle tenderness and Chronic pain; whereas in Myofascial Pain, Regional muscle tenderness exists associated with and pain for a short period.

Recent studies using more sophisticated measures of experimental pain testing, gave important information on FM that these patients experience tenderness everywhere in the body, and not just only at the mentioned tender points. This made researchers conduct subsequent studies on FM [9].

Revelation of multiple potential mechanisms, that may be responsible for pain amplification in FM has been proposed. Few among them are a decrease in the activity of descending analgesic pathways, an increase in central pain facilitatory pathway, and a diffuse increase in all peripheral sensory stimuli processing.

Investigatory studies have revealed that there is an evidence of attenuated dopaminergic activity in FM patients, with a consequent decrease in the availability of $\mu$ - opioid receptors for the binding of opioid like molecules, which would otherwise help in analgesia. It is also said that the decrease in $\mu$ - opioid receptors is due to their increased binding by endogenous opioid production in FM patients [6].

Hence changes in the central nervous system is thought to be the absolute dominant in the FM studies along with peripheral factors which also play an important role in the pathogenesis and treatment of the same.

Central pain processing is said to be co-opted to the peripheral nociceptive stimuli having a synergetic influence on each other. 
Certain peripheral conditions like MPS, TMDs, osteoarthritis can herald their noxious afferent stimuli exaggerating hyperalgesia seen in FM. Consequent to this if these peripheral conditions are treated, there could be alleviation of FM pain to certain extent [5].

So, patients with comorbid conditions causing ongoing peripheral nociceptive input would potentially benefit from treatments aiming the reduction of peripheral drive of central sensitization. Researches are on to counter the effects of LPC 16:0 by administering Darapladib, a selective Platelet Activating Factor-Acetyl Hydrolase Inhibitor, which degrades phospholipids, thereby blocking the production of LPC. Antioxidants too had a similar benefit [4].

\section{Conclusion}

Among the biomarkers like Serotonin,5HT2A receptors, Serotonin transporter, Dopamine 4 receptor, Protein backbones and Pyridine Carboxylic acid, that were found to be significant in causing FM, the presence of LPC 16:0 was the most recent molecule found directly related to stress causing FM. Although LPC has been found to be a very significant molecule towards causing FM, further studies, researches, and clinical trials need to be carried out in this regard in order to enhance the significance, specificity and sensitivity of this molecule towards diagnosing and completely treating Fibromyalgia.

\section{Bibliography}

1. Galvez-Sánchez CM and Reyes Del Paso GA. "Diagnostic Criteria for Fibromyalgia: Critical Review and Future Perspectives". Journal of Clinical Medicine 9.4 (2020): 1219.

2. Hung $\mathrm{CH}$., et al. "Activation of acid-sensing ion channel 3 by lysophosphatidylcholine 16:0 mediates psychological stressinduced fibromyalgia-like pain". Annals of the Rheumatic Diseases 79.12 (2020): 1644-1656.

3. Hackshaw KV. "The Search for Biomarkers in Fibromyalgia". Diagnostics (Basel) 11.2 (2021): 156.

4. Hackshaw KV., et al. "Metabolic fingerprinting for diagnosis of fibromyalgia and other rheumatologic disorders". Journal of Biological Chemistry 294.7 (2019): 2555-2568.

5. Arnold LM., et al. "AAPT Diagnostic Criteria for Fibromyalgia". Journal of Pain 20.6 (2019): 611-628.

6. Hackshaw KV., et al. "Metabolic fingerprinting for diagnosis of fibromyalgia and other rheumatologic disorders". Journal of Biological Chemistry 294.7 (2019): 2555-2568. Erratum in: J Biol Chem. 2020 Apr 24;295 (17): 5834.
7. Häuser W and Fitzcharles MA. "Facts and myths pertaining to fibromyalgia”. Dialogues in Clinical Neuroscience 20.1 (2018): 53-62.

8. Malatji BG., et al. "A diagnostic biomarker profile for fibromyalgia syndrome based on an NMR metabolomics study of selected patients and controls". BMC Neurology 17.1 (2017): 88.

9. Üçeyler N., et al. "Ätiologie und Pathophysiologie des Fibromyalgiesyndroms : Aktualisierte Leitlinie 2017 und Übersicht von systematischen Übersichtsarbeiten sowie eine Übersicht über Studien zur Kleinfaserpathologie bei Subgruppen des FMS [Etiology and pathophysiology of fibromyalgia syndrome : Updated guidelines 2017, overview of systematic review articles and overview of studies on small fiber neuropathy in FMS subgroups]". Schmerz 31.3 (2017): 239-245.

10. Clauw DJ. "Fibromyalgia: a clinical review". JAMA 311.15 (2014): 1547-1555.

11. Clauw DJ. "Fibromyalgia: an overview". American Journal of Medicine 122 (2009): S3-S13.

\section{Volume 5 Issue 12 December 2021 (C) All rights are reserved by Gaurav., et al.}

